\title{
Time-Dependent Bactericidal Efficacy of Hydrogen Peroxide against Methicillin-Resistant Staphylococcus Aureus and Acinetobacter Baumannii in Second Degree Burn Wound
}

\author{
Abidullah Khan ${ }^{1}$, Guohui Xiao ${ }^{2}$, Chuangang You ${ }^{1}$, Amin Khan ${ }^{3}$ and Chunmao Han*1 \\ ${ }^{1}$ Department of Burns, Second Affiliated Hospital of Zhejiang University, School of Medicine, Hangzhou 310009, China
}

${ }^{2}$ Department of Microbiology, Zhejiang University, School of Medicine, Hangzhou 310009, China

${ }^{3}$ Department of Chemistry, University of Science and Technology, Bannu, Khyber Pakhtunkhwa (KPK) 28100, Pakistan

*Corresponding author: Chunmao Han, Department of Burns, Second Affiliated Hospital of Zhejiang University, School of Medicine,

Jiefang Road 88, Hangzhou 310009, China

\section{ARTICLE INFO}

Received: 蔧 February 18, 2019

Published: 慧 March 06, 2019

Citation: Abidullah Khan, Guohui Xiao, Chuangang You, Amin Khan, Chunmao Han. Time-Dependent BactericidalEfficacy of Hydrogen Peroxide against MethicillinResistant Staphylococcus Aureus and Acinetobacter Baumannii in Second Degree Burn Wound. Biomed J Sci \& Tech Res 15(4)-2019. BJSTR. MS.ID.002720.

Keywords: Hydrogen Peroxide; Antimicrobial; Mrsa; Acinetobacter Baumannii; Infection; Burn Wound; Mice

\begin{abstract}
Nosocomial infections are major health care problem and their developing antibiotics resistance have made it harder to treat. Hydrogen peroxide $\left(\mathrm{H}_{2} \mathrm{O}_{2}\right)$ is widely used for contaminated wound sterilization, but its efficacy and time of sterilization against MethicillinResistant Staphylococcus aureus (MRSA) and Acinetobacter baumannii (A. baumannii) in burn wound has yet to be fully determined. In this study, we investigated the time-dependent bactericidal activity of $3 \%$ hydrogen peroxide. In vitro studies, MRSA and A. baumannii treated with hydrogen peroxide for $30 \mathrm{sec}$ led to $100 \%$ bacterial kill. For in vivo studies, second degree burns was created on the back of mice followed by inoculation with $1 \times 10^{8} \mathrm{CFU}$ ml-1 of MRSA and A. baumannii, isolated from clinical specimens of burn patients. Hydrogen peroxide application efficiently reduced bacterial burden $6.9 \times 10^{5} \mathrm{CFU} / \mathrm{g}$ (control: $7.5 \times 10^{10}$ $\mathrm{CFU} / \mathrm{g}$ ) for $30 \mathrm{sec}$ to $3.2 \times 10^{4} \mathrm{CFU} / \mathrm{g}$ (control: $9.2 \times 10^{9} \mathrm{CFU} / \mathrm{g}$ ) for $120 \mathrm{sec}$ of application duration. The $\log 10$ CFU bacterial load reduction with hydrogen peroxide application from $30 \mathrm{sec}$ to $120 \mathrm{sec}$ was found to be $0.96(\mathrm{P}<0.001)$. Furthermore, histological evaluation of the hydrogen peroxide $(3 \%)$ treated and untreated burn wounds did not exhibit any significant tissue alterations. These results emphasize the potential use of $3 \%$ hydrogen peroxide as a possible treatment option for the decolonization of MRSA and A. baumannii bacteria in burn wounds without harming the tissue viability.
\end{abstract}

\section{Introduction}

Burns are common and major healthcare problem that requires care in specialized burns units [1]. The nature and extent of burn injuryleads to immunosuppression in patients, resulting inincreased susceptibility to various nosocomial pathogens [2]. MRSA accounts for $11.5 \%$ of the total $S$. aureus isolates from burn wound patients. Studies have shown that the prevalence of infections caused by MRSA has increased from $12 \%$ in 1992 to $80.03 \%$ in 2012 [3]. Many MRSA isolates are becoming multidrug-resistantand are susceptible to only tetracyclines and glycopeptides [4]. The commonly deployed antibiotics to treat MRSA mediated burn infections (tigecycline, vancomycin) are associated with several side effects. Also, S. aureus strains resistant to these antibiotics have already emerged in hospital and community settings [5]. A. baumannii is a member of opportunistic bacteria and capable of developing drug resistance and biofim formation [6]. The only available antibiotic treatments possesses additional damage to the patients [7]. Thus, a key challenge is to use simple, low-cost and effective treatments to develop non-antibiotic therapies to prevent MRSA and A. baumannii colonization in burn wounds.

One method for reducing wound bacterial load and colonization is the use of topical antiseptics agents. The advantage of antiseptics is that they are less selective in their action and bacteria are less 
likely to develop resistance [8]. Hydrogen peroxide (3\%) exhibits broad antimicrobial activity in vitro [9] and in vivo [10]. Studies have been conducted to observe the antimicrobial activity of hydrogen peroxide and its effect on wound healing. A randomized clinical trial of burn wounds showed that wounds treated with $2 \%$ hydrogen peroxide significantly improved skin graft take rates [11]. Another clinical trial demonstrated that hydro surgery with hydrogen peroxide was superior to controls without hydrogen peroxide for infected wounds, resulting in shorter hospital stay and enhanced graft viability [12]. The clinical use of hydrogen peroxide regarding the elimination of MRSA and A. baumannii is limited. Therefore, a better understanding of hydrogen peroxide in sterilization and its impact on the skin will enhance its potential use. In this paper, we studied the use of $3 \%$ hydrogen peroxide as one adjunct for reducing MRSA and A. baumannii burden in burn wound. We investigated time - dependent antimicrobial activity of hydrogen peroxide by creating the mice burn model infected with MRSA and A. baumannii. We also observed histological alterations in burn wound upon hydrogen peroxide application.

\section{Materials and Methods}

\section{Materials}

Tryptic soy broth (TSB), Luria-Bertani (LB) broth, Phosphate buffered saline (PBS), Mueller-Hinton (MH) agar and 3\% hydrogen peroxide was purchased from Bainafo Bioengineering Co. Ltd (Zhengzhou, China). MRSA and A. baumannii bacteria strains were obtained from the Department of Clinical Microbiology, Second Affiliated Hospital of Zhejiang University.

\section{Experimental Animals}

Pathogen-free BLAB/c mice (18-25 grams; 7-8 weeks of age) were purchased from Nanjing Biomedical Research Institute of Nanjing University (Nanjing, China). The mice were allowed to acclimate in the animal facility for ten days with free access to food and water. Animals were divided into 8 groups ( $n=6 /$ group) based on the hydrogen peroxide application duration. All procedures performed on mice were in accordance with the Guidelines for Animal Experimentation of Zhejiang University.

\section{Antibacterial Activity by Disc Diffusion Method}

The antimicrobial activity of hydrogen peroxide was performed using standard techniques. MRSA and A. baumannii separately cultured overnight was sub-cultured for $4 \mathrm{~h}$. $50 \mu \mathrm{l}$ aliquots of freshly prepared MRSA and A. baumannii suspension in PBS at optical density of OD $600=0.1$, were seeded onto the MH agar plate. Sterile filter discs (5 $\mathrm{mm}$ ) were impregnated with $3 \%$ hydrogen peroxide (PBS was used as a control) and incubated at $37{ }^{\circ} \mathrm{C}$ for 24 h. Antimicrobial activity was evaluated by measuring the diameter of bacteria inhibitory zone.

\section{MRSA and A. Baumannii Treatment with Hydrogen Peroxide}

MRSA ( $\left.1 \times 10^{8} \mathrm{CFU} \mathrm{ml}-1\right)$ and A. baumannii $\left(1 \times 10^{8} \mathrm{CFU} \mathrm{ml}-\right.$ $1)$, suspensions in LB broth were centrifuged and rinsed twice with PBS. MRSA and A. baumannii pellets were then subjected to $100 \mu \mathrm{l}$ of $3 \%$ hydrogen peroxide at predetermined time $(30 \mathrm{sec}, 60 \mathrm{sec}$, $90 \mathrm{sec}$ and $120 \mathrm{sec}$ ). Bacterial suspension treated with hydrogen peroxide for various time periods was immediately introduced in $45 \mathrm{ml}$ of PBS (reaching hydrogen peroxide final concentration to $0.001 \%$ ) and centrifuged. Control group was treated the same, but with sterile PBS. After centrifugation, the supernatant was discarded, and bacteria were resuspended in $1 \mathrm{ml}$ PBS. Aliquot of $50 \mu \mathrm{l}$ were plated on $\mathrm{MH}$ agar and incubated at $37^{\circ} \mathrm{C}$ for $24 \mathrm{~h}$.

\section{Creation of burn wound and MRSA/ A. Baumannii Infection}

Mice dorsal surface was shaved and depilated with Veet (Reckitt Benckiser, China) 1 day before creation of burn wound. The following day, mice were anaesthetized and $1.5 \mathrm{~cm}$ diameter partial thickness burn $\left(80^{\circ} \mathrm{C}\right.$ for $\left.6 \mathrm{sec}\right)$ was created using burn creation machine (ZH-YLS-5Q China) (Figure 1). This method was based on the protocol by Liu and colleagues [13]. Soon after, mice were resuscitated with $1 \mathrm{ml}$ sterile saline injected intraperitoneally. $20 \mathrm{~min}$ after the creation of the burn (allowing the burn injury to cool down), elastic bandage tape was applied to burn wound and stripped from $(1.5 \times 1.5 \mathrm{~cm})$ skin area, 8 times in one direction and 8 times in the opposite direction, until the skin became reddened with no apparent bleeding. This method is based on protocols by Tatiya et al [14]. This method allows bacteria to colonize in the wound. A $15 \mu$ l MRSA/ A. baumannii $\left(1 \times 10^{8} \mathrm{CFU}\right.$ ml-1) suspension in PBS was smeared onto the burn wound with an inoculating loop. Burn wound was then covered with polyurethane film (Tegaderm, Shanghai, China) for $24 \mathrm{~h}$ to mimic a stable bacterial infection.

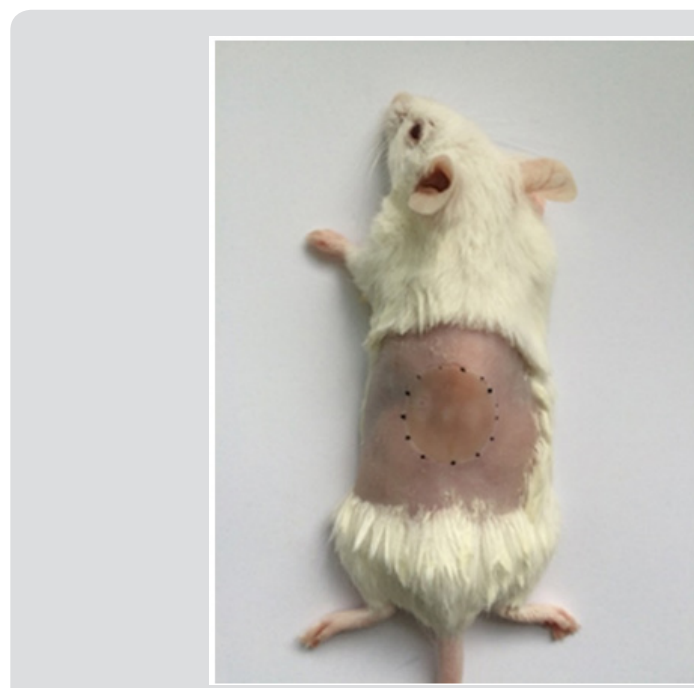

Figure 1: Mice burn model. 
Treatment of Burn Infection and Microbiological Analysis

$24 \mathrm{~h}$ after bacterial seeding onto the burn wound, it was treated with $2-5 \mathrm{ml}$ of $3 \%$ hydrogen peroxide for duration of $30 \mathrm{sec}, 60 \mathrm{sec}$, $90 \mathrm{sec}$ and $120 \mathrm{sec}$, followed by a rinse with PBS. Mice in the control group were treated the same, but with sterile PBS. Mice were sacrificed, and wound tissue samples were excised using a punch skin biopsy (Skalr, USA). Samples were then homogenized in PBS and 10 -folds serially diluted bacterial colonies were enumerated by plating on MH agar.

\section{Hydrogen Peroxide Effect on The Burn Wound Histology}

The effect of hydrogen peroxide on the burn wound was evaluated by hematoxylin and eosin (H \& E) staining. Briefly, $24 \mathrm{~h}$ after the creation of burn wound, it was subjected to $3 \%$ hydrogen peroxide for $120 \mathrm{sec}$ followed by a rinse with PBS. Control group was treated the same, but with PBS. Tissue samples $(5 \times 5 \mathrm{~mm})$ were excised, fixed in buffered formalin (10\%), and dehydrated with al- cohol and finally embedded in paraffin wax into blocks. Sections of tissue samples were stained with H\&E and examined under a light microscope (Nikon, Japan).

\section{Statistical Analysis}

Data were presented as means \pm standard deviation. Statistical analysis was executed using GraphPad Prism 5.0 (GraphPad Software, La Jolla, CA, USA) P-values were determined using twotailed Student's t-test. $\mathrm{P}<0.05$ were considered to be significant difference.

\section{Results}

\section{In Vitro Antimicrobial Activity of Hydrogen Peroxide}

Hydrogen peroxide application showed a promising antimicrobial activity. Zone of inhibition was calculated to be $30 \mathrm{~mm}$ (MRSA) and $28 \mathrm{~mm}$ (A. baumannii) compared with control group (Figure 2). Also, MRSA and A. baumannii treated with hydrogen peroxide for $30 \mathrm{sec}$ led to $100 \%$ bacterial kill in comparison with the control group (Figure 3).
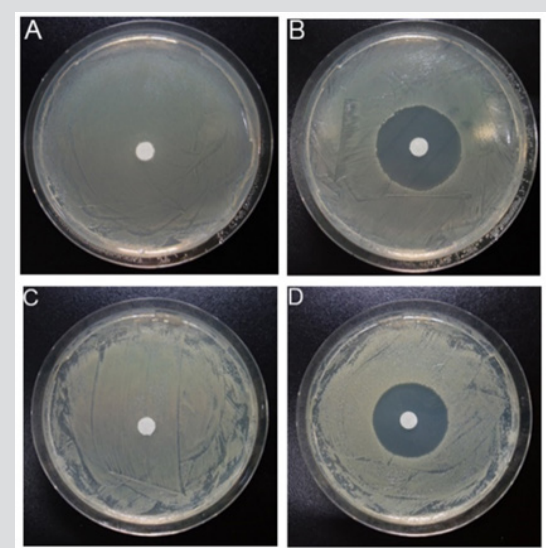

Figure 2: Antibacterial activity of 3\% hydrogen peroxide. Top: halos representing zone of inhibition of MRSA (A) Control and (B) 3\% hydrogen peroxide treated. bottom: halos representing A. Baumannii zone of inhibition, (C) Control (PBS) and (D) 3\% hydrogen peroxide treated.
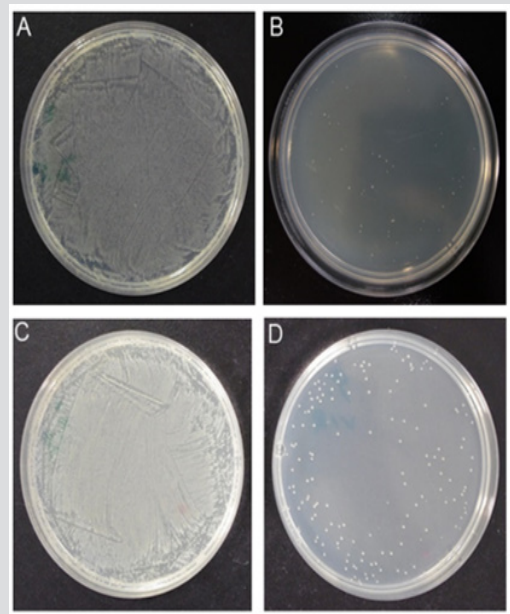

Figure 3: The antibacterial activity of 3\% hydrogen peroxide. Top: (A) MRSA treated with PBS for 30 sec, (B) MRSA treated with hydrogen peroxide for $30 \mathrm{sec}$. bottom: (C) A. baumannii treated with PBS for $30 \mathrm{sec}$, (D) A. baumannii treated with hydrogen peroxide for $30 \mathrm{sec}$. 


\section{In Vivo Antimicrobial Activity of Hydrogen Peroxide}

The antibacterial activity of $3 \%$ hydrogen peroxide against MR$\mathrm{SA} / A$. baumannii was determined by measurements of bacterial load in burn wound. Application of hydrogen peroxide for specified duration (30 sec, $60 \mathrm{sec}$, $90 \mathrm{sec}$ and $120 \mathrm{sec}$ ) suppressed MRSA/ A. baumannii load in a time-dependent manner. Hydrogen peroxide application for $30 \mathrm{sec}$ reduced $6.9 \times 10^{5} \mathrm{CFU} / \mathrm{g}$ bacterial load compared with control group $\left(7.5 \times 10^{10} \mathrm{CFU} / \mathrm{g}\right)$. The $\log 10 \mathrm{CFU}$ bacterial reduction was found to be $5.07(\mathrm{P}<0.0001)$. Furthermore, increase in hydrogen peroxide application time further reduced the bacterial load, i.e. $60 \mathrm{sec}\left(4.9 \times 10^{5} \mathrm{CFU} / \mathrm{g}\right.$ vs. control $1.21 \mathrm{x}$ $\left.10^{10} \mathrm{CFU} / \mathrm{g}\right) \log 10 \mathrm{CFU}$ bacterial reduction of 4.39 ( $\left.\mathrm{P}<0.0001\right), 90$ $\sec \left(8.20 \times 10^{4} \mathrm{CFU} / \mathrm{g}\right.$ vs. control $\left.1.61 \times 10^{10} \mathrm{CFU} / \mathrm{g}\right) \log 10 \mathrm{CFU}$ bacterial reduction of $5.30(\mathrm{P}<0.0001)$, and $120 \mathrm{sec}\left(3.2 \times 10^{4} \mathrm{CFU} / \mathrm{g}\right.$ vs. control $\left.9.2 \times 10^{9} \mathrm{CFU} / \mathrm{g}\right) \log 10 \mathrm{CFU}$ bacterial reduction of 6.03 $(\mathrm{P}<0.0001)$. The total bacteria $\log 10 \mathrm{CFU}$ reduction with hydrogen peroxide application from $30 \mathrm{sec}$ to $120 \mathrm{sec}$ was found to be 0.96 $(\mathrm{P}<0.001)$ (Figure 4). These results show, increasing hydrogen peroxide application time can further reduce wound bacterial load.

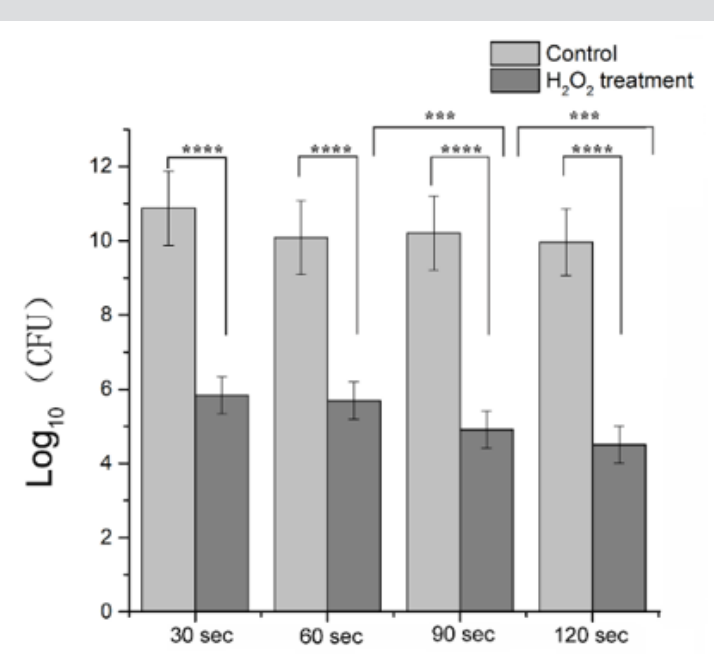

Figure 4: Antimicrobial effect of 3\% hydrogen peroxide on MRSA and A. baumannii in burn wound. Bacterial counts were calculated by plating serially diluted homogenate onto agar plates and incubated at $37^{\circ} \mathrm{C}$ for $24 \mathrm{~h}$. ${ }^{* * *} \mathrm{P}<0.0001,{ }^{* * *} \mathrm{P}<0.001$.
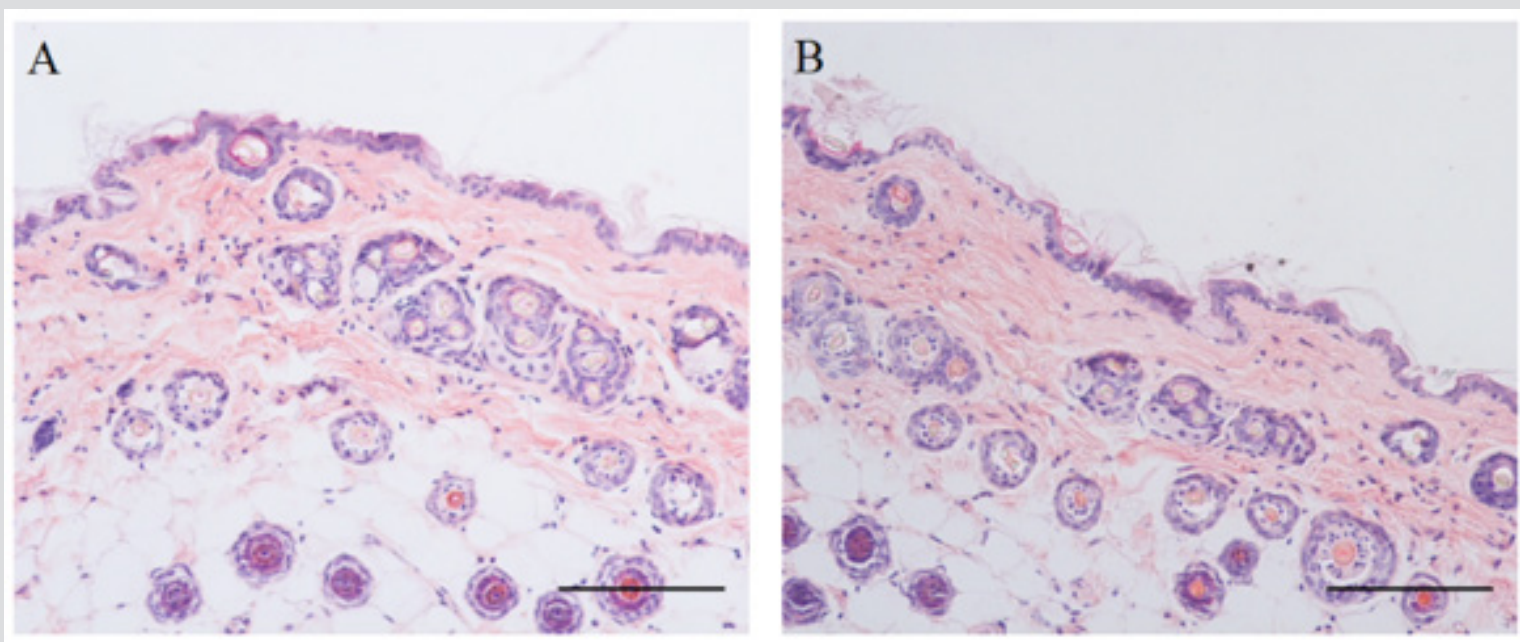

Figure 5: The histological analysis of mice skin treated with 3\% hydrogen peroxide for 120 sec. Haematoxylin and eosin staining of (A) Control (PBS) and (B) hydrogen peroxide treated (magnification 100x; scale bar $100 \mu \mathrm{m}$ ).

\section{Hydrogen Peroxide Effect on Burn Wound}

Histological studies of burn wound tissue treated with hydrogen peroxide for $120 \mathrm{sec}$ revealed no significant difference compared to PBS treated control group. Congestion and edema were absent in both hydrogen peroxide and control treated groups (Figure 5).

\section{Discussion}

In this study, mice burn wounds infected with MRSA and $A$. baumannii were used to evaluate the antimicrobial effect of $3 \%$ hydrogen peroxide in time-dependent manner. MRSA and $A$. baumannii bacteria were smeared on burn wound, and $24 \mathrm{~h}$ later 
a stable skin infection was created to mimic infected burn wound. Our results demonstrated reduction of bacterial load was based on the duration of hydrogen peroxide application and contact with bacteria. Histological evaluation of burn wound treated with hydrogen peroxide for $120 \mathrm{sec}$ has no apparent changes when compared with the control group, showing that hydrogen peroxide did not alter the tissue viability.

Wound cleaning and sterilization are crucial for management of traumatic wounds to minimize the rate of infection [15]. Burn wounds especially the deep partial and full thickness burns consist of avascular necrotic tissue (eschar) providing a protein-rich environment for bacterial colonization and proliferation [1].

Antiseptics such as ethanol, triclocarban, chlorhexidine, chlorine, silver and iodine compounds destroy or inhibits the growth and development of bacteria and carries broader antimicrobial activity than antibiotics. Hydrogen peroxide is a well-known antiseptic, and $3 \%$ solution shows a broad spectrum of antimicrobial activity [9]. In our in vitro study, both gram-positive (MRSA) and gram-negative (A. baumannii) bacteria treated with $3 \%$ hydrogen peroxide for $30 \mathrm{sec}$ resulted in 100\% kill (Figure 3). This result shows that $3 \%$ hydrogen peroxide can be used for sterilization of burn and ICU units to effectively eliminating gram - positive and gram-negative bacteria, thus reducing the rate of nosocomial infections. The use of antiseptics increases the rate of wound healing by minimizing and preventing infection of open wounds [16]. Another argument about the use of antiseptics is to prevent bacterial resistance developed by the use of antibiotics [17]. In our in vivo study, $3 \%$ hydrogen peroxide efficiently reduced both MRSA and A. baumannii, which are difficult to treat with intravenous or topical antibiotics. Antiseptics reduce the use of antibiotics in burn patients. The presence of catalases in tissue can make the bactericidal activity of hydrogen peroxide less effective in vivo [18].

In our study, we concluded the hydrogen peroxide effect of reducing bacterial load is time dependent manner. Burn wound treated hydrogen peroxide for $120 \mathrm{sec}$ significantly reduced bacterial load, thus reducing the risk of sepsis and biofilm formation (Figure 4). Also, we should keep in consideration that further increase in hydrogen peroxide application didn't reduce bacteria which might be associated with bacteria being penetrated deep into the wound and could not come in contact with hydrogen peroxide. Studies conducted on human and animals have shown that hydrogen peroxide has no negative impact on wound healing [19]. In a combined in vitro study and clinical trial, hydrogen peroxide accelerates wound repithelialization [20]. In our study, the application of 3\% hydrogen peroxide did not exhibit any alterations in tissue histology after a brief exposure of $120 \mathrm{sec}$ (Figure 5). Hydrogen peroxide did not cause any immediate tissue damage, which might be associated with the hydrogen peroxide dilution in burn wound or short duration of application.

\section{Conclusion}

In Conclusion, $3 \%$ hydrogen peroxide is an effective antiseptic agent, which can reduce MRSA and $A$. baumannii related burn wound infection and sepsis. Hydrogen peroxide effectiveness increases with increase in application time without harming the tissue viability. However, clinical studies need to be conducted to evaluate its efficacy on infected burn wounds.

\section{Acknowledgement}

This work was financially supported by the National key research and development project (2016YFC1100800), and the National Natural Science Foundation of China (81671909, 81772069) and Natural Science Foundation of Zhejiang Province, China (LY15H150004, LQ16H150002).

\section{References}

1. D Church, S Elsayed, O Reid, B Winston, R Lindsay, et al. (2006) Burn wound infections. Clin Microbiol Rev 19(2): 403-434.

2. U Altoparlak, S Erol, MN Akcay, F Celebi, A Kadanali (2004) The timerelated changes of antimicrobial resistance patterns and predominant bacterial profiles of burn wounds and body flora of burned patients. Burns 30(7): 660-664.

3. D Raghunath (2008) Emerging antibiotic resistance in bacteria with special reference to India. J Biosci 33(4): 593-603.

4. JJ Ruhe, A Menon (2007) Tetracyclines as an oral treatment option for patients with community onset skin and soft tissue infections caused by methicillin-resistant Staphylococcus aureus. Antimicrob Agents Chemother 51(9): 3298-303.

5. GE Stein, WA Craig (2006) Tigecycline: a critical analysis. Clin Infect Dis 43(4): 518-524.

6. P Scott, G Deye, A Srinivasan, C Murray, K Moran, et al. (2007) An outbreak of multidrug-resistant Acinetobacter baumannii-calcoaceticus complex infection in the US military health care system associated with military operations in Iraq. Clin Infect Dis 44(12): 1577-1584.

7. DP Crane, K Gromov, D Li, K Soballe, C Wahnes, et al. (2009) Efficacy of colistin-impregnated beads to prevent multidrug-resistant A. baumannii implant-associated osteomyelitis. J Orthop Res 27(8): 1008-1015.

8. AD Russell (2002) Antibiotic and biocide resistance in bacteria: introduction. J Appl Microbiol 92: 1-3.

9. G McDonnell, AD Russell (1999) Antiseptics and disinfectants: activity, action, and resistance. Clin Microbiol Rev 12(1): 147-79.

10. CD Brown, JA Zitelli (1993) A review of topical agents for wounds and methods of wounding. Guidelines for wound management. J Dermatol Surg Oncol 19(8): 732-737.

11. AA Mohammadi, SM Seyed Jafari, M Kiasat, MR Pakyari, I Ahrari, et al. (2013) Efficacy of debridement and wound cleansing with $2 \%$ hydrogen peroxide on graft take in the chronic-colonized burn wounds: a randomized controlled clinical trial. Burns 39(6): 1131-1136.

12. S Irkoren, N Sivrioglu (2014) A hydrosurgery system (Versajet) with and without hydrogen peroxide solutions for the debridement of subacute and chronic wounds: a comparative study with hydrodebridement. Adv Skin Wound Care 27(3): 127-131.

13. Y Liu, X Zhang, Z Zhang, PY Fang, WS Xu, et al. (2004) [Effects of topical application of insulin on the wound healing in scalded rats]. Zhonghua Shao Shang Za Zhi 20(2): 98-101.

14. N Tatiya-Aphiradee, $W$ Chatuphonprasert, K Jarukamjorn (2016) In vivo antibacterial activity of Garcinia mangostana pericarp extract against 
methicillin-resistant Staphylococcus aureus in a mouse superficial skin infection model. Pharm Biol 54(11): 2606-2615.

15. MN Khan, AH Naqvi (2006) Antiseptics, iodine, povidone iodine and traumatic wound cleansing. J Tissue Viability 16(4): 6-10.

16. G Dow, A Browne, RG Sibbald (1999) Infection in chronic wounds: controversies in diagnosis and treatment. Ostomy Wound Manage 45(8): 23-27.

17. AS Colsky, RS Kirsner, FA Kerdel (1998) Analysis of antibiotic susceptibilities of skin wound flora in hospitalized dermatology patients.
The crisis of antibiotic resistance has come to the surface. Arch Dermatol 134(8): 1006-1009.

18. A Drosou, A Falabella, RS Kirsner (2003) Antiseptics on Wounds: An Area of Controversy. Wounds A Compendium of Clinical Research \& Practice 15(5): 149-166.

19. W Lineaweaver, R Howard, D Soucy, S McMorris, J Freeman, et al., (1985) Topical antimicrobial toxicity. Arch Surg 120(3): 267-270.

20. RP Gruber, L Vistnes, R Pardoe (1975) The effect of commonly used antiseptics on wound healing. Plast Reconstr Surg 55(4): 472-476.

\section{ISSN: 2574-1241}

DOI: 10.26717/BJSTR.2019.15.002720

Chunmao Han. Biomed J Sci \& Tech Res

(c) (P) This work is licensed under Creative

Submission Link: https://biomedres.us/submit-manuscript.php

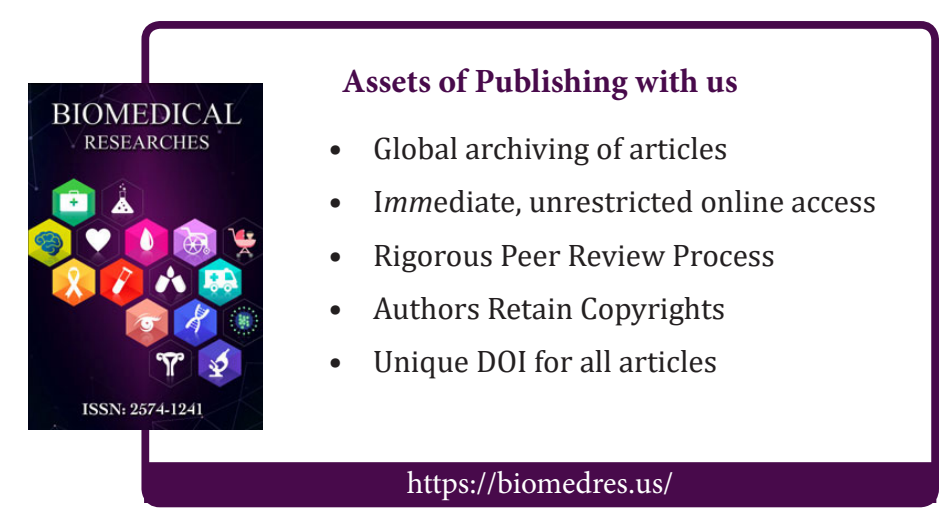

